\title{
An Analysis of Rumors Spread in Macao
}

\author{
Kang, Jiali ${ }^{1, a}$ \\ ${ }^{1}$ The Institute for Social and Cultural Research (ISCR), Macao University of Science \& Technology, \\ Taipa, Macao SAR, China \\ ame@mail.im
}

Keywords: Rumor; Spread; Social governance

\begin{abstract}
With the influence of various factors, the impact of rumors on Macao's society has shown an expanding trend along the development of the times. This article begins with an exploration of the nature and characteristics of the rumors. After analyzing the structure of the rumors, it introduces the unique geographical and social characteristics of Macao and attempts to analyze the path of rumors spread by using the characteristics in combination with recent rumors which are significant influence in Macao. Finally, it provides some constructive solutions.
\end{abstract}

\section{浅析谣言在澳门的传播 \\ 康 佳 $^{\text {立 }}{ }^{1, a}$ \\ 1 澳门科技大学, 函仔, 澳门, 中国 \\ ame@mail.im}

关键词：谣言; 传播; 社会治理

摘 要: 伴随着众多因素的作用, 谣言对澳门社会的影响力随着时代的发展呈现扩大的趋势。 本文从探讨谣言的本质和特征入手, 在剖析了谣言的结构后, 引入了澳门本地独特的地理和 社会特征, 尝试通过利用这种特征结合近期澳门本地具有重大影响的谣言事件, 来分析谣言 在澳门的传播轨迹。最后由此提供一些具有建设性的解决方式。

\section{1. 对谣言的定义和普遍特征的认定}

谣言, 是汉语中的一个常用词汇, 古已有之。同时它也是一种广泛存在于社会之中的文 化现象，不会因时代、群体和社会阶层的不同而销声匿迹。《现代汉语词典》认为其是 “没有 事实根据的消息”。《辞海》的解释是：“谣，是没有根据的传闻或凭空捏造的话; 谣言，是没 有事实根据的传闻或捏造的消息”。《韦伯斯特英文大字典》中则解释成 “谣言是一种缺乏真 实根据, 或未经证实, 公众一时难以辨别真伪的闲话、传闻或與论”。只是无论对谣言解释的 程度有多么繁杂, 均突出了它的两个核心要素, 即谣言的形式为某些在传播过程中的言语或 消息; 谣言的性质是无法辨别真实性。通过这两个核心要素的把握, 能够对 “谣言” 的本质 有清晰的了解，同时可以对谣言的特征加以认定。

谣言最重要的特征是受众强烈关注的信息, 多为与其密切相关的事件, 它是谣言产生的 源泉。正因为这类信息关注程度高, 公众对信息的需求也高。除了能够引领人们的兴趣外, 有利于其在人群中的传播, 也更容易让人接受谣言并进行下一次的传播, 为谣言的传播提供 了重要的推手，使其传播的速度加快。

其次，传播是谣言的生命力，谣言的存在首先是运动的结果。这一运动表现在信息的传 播上，使其本质上为虚假信息的内容开始扩散成谣言。在谣言停止传播之时，也是对其的终 结, 谣言本身又回归到最初的谣言内容的状态, 即虚假信息。所以，对谣言的描述，从来应 
该是对一种持续传播的虚假信息的描述。同时谣言的传播在范围和速度上与其存在的时长成 正比。即传播越快的谣言, 波及面越广, 同时存在的时间越长, 影响力越大, 给谣言的消除 带来了巨大的干扰，这反而提高了谣言的生命力。

谣言传播的条件是需要获得受众的信任并被接受。它为谣言在受众间的传播提供了桥梁 的作用，使得谣言能够持续的传播下去。因为一旦传播中的虚假信息被 “证伪”，即被认定为 “谣言” 后，受众对虚假本质的接受程度决定了谣言是否能被继续传播，这也决定了谣言的 生命。毕竟受众除了会对已经被证伪信息的虚假性质感兴趣外，并不会本能的去传播已然被 认定为谣言的信息。除非他们有意作为谣言的源头，而非是受众来推动谣言的传播。

再次，谣言所具有的普遍性和扁平化特征在扩大了受众面的同时，成为其在人际间传播 的土壤。它重点表明了受众对谣言的认知具有群体性, 即同一社群对单一谣言的认知往往要 大于不同社群，并在传播过程中具有直接和快速的效果。

然而快速传播的信息带来的一个显著特征就是传播过程中的损耗。这种损耗有无意和有 意两种区分, 谣言也不例外。无意的损耗也是自然的损耗, 任何信息在传播过程中无法避免。 而有意的损耗是在传播过程中，对信息加以歪曲、修改或增加冗余等手段来达成一定目的， 迫使其发生人为设定的改变的行为。损耗往往造成的信息不对称又加剧了谣言在传播过程中 的裂变，于是老谣言也是新谣言诞生的母体，新谣言总是伴随着老谣言而出现。

18 世纪苏格兰哲学家，素称 “怀疑主义”之父的大卫・休谟（David Hume，1711-1776） 在《人类理解力研究》一书中, 提出了理性思维总原则的 “休谟公理”, 大意是人们不应该倾 向接受没有足够证据支撑的事件。而谣言的模糊性特征，正好验证了受众在获得信息时的反 应与“休谟公理”相反，而这类信息往往在事后被认定为谣言。

这里的模糊需要从以下两个方面去了解, 即:

难以界定。谣言往往无法提供详实的事例和数据来支撑, 本质上是一种可供人们消费的 快餐性信息，迎合了人们不需要进行费时费力研判等等的本性。

难以纠责。谣言的基本特征使谣言本身很难界定其造成的危害以及所应承担责任的负责 人。正是因为这种 “冊须负责” 或责任感较低的特点，让谣言在普通环境下的产生和传播几 乎没有代价。在这一层面看来, 需要达成一定目的而故意造谣的传播源头, 几乎就是零成本 代价而获利巨大。其一旦在监管薄弱的情况下出现, 更加速了谣言的传播, 加重了对社会的 危害。[1]

另外任何传播中的谣言，包括 “戏谑”、“开玩笑” 在内，不论其目的是否有实际的获利 产生，均为有一定目的的存在。之所以被认定是 “谣言”，是经过传播过程后的被动结果。对 其虚假性质的认定所具有的延迟，是建立在达到传播源头一定的目的并产生了影响之后的结 果。因此, 任何谣言都是有结果产生的虚假消息, 已经产生了或多或少的影响。这是谣言的 价值特征。

简单化也是谣言的基本特征之一。信息的繁简程度与传播的速度成正比，即越简单的信 息传播速度越高传播得越快，效率也越高。谣言的负面性是建立在对群体社会性的解构过程 之中, 它需要尽可能的简单化来获得快速传播加大影响力, 同时接触更多的组织机构和社会 层级, 获得更长久的生命力。此外这种被简单化的谣言加速传播带来的损耗, 给新谣言的出 现提供了更多可以操作的空间。这类新谣言多是在简单化谣言的基础上出现, 本质上是对简 单化谣言的增补在达到一定程度后的谣言本体发生改变，它为谣言的裂变提供了基础。

只是单一谣言存在的目的性, 使其快速的传播不能以牺牲谣言本身的价值为代价, 它需 要保证本体的完整和与本体相关的重要信息的存在。因此在本体不变的情况下, 对其的解释 充当了谣言的“保护膜”, 它即保护了谣言的内核一一本体, 又使谣言区别于其它可信的信息, 是谣言的内容所在是其存在的关键因素。

同时，谣言的内容实质上是对其指向事件的表述。这种表述是客观存在的现实，与谣言 共生共灭，是谣言的组成部分。在其传播过程中不会因为表述内容的真实性而受到影响。可 
以说, 谣言的内容是静止的、单一的客观存在, 并不具有谣言的 “传播” 特征。谣言的内容 需要经过传播才能真正意义上成为 “谣言”, 才具有谣言一系列的特征和影响力, 毕竟传播是 谣言的生命力, 谣言在本质上从来都是运动的状态。

由此衍生出了谣言的基本结构，即：

$$
\text { 谣言=传播中的虚假信息 }
$$

虚假信息 (即谣言内容) $=$ 表述事件的本体 + 对本体的解释

从中可以看到谣言本质上是和传播、事件的本体以及对其的解释相关。其中任何谣言的 本体也是谣言事件的本体, 它是谣言的核心, 是辨别不同谣言的标志。因此同一谣言中的本 体往往是恒定的状态, 其多由受众身边重要的人、物或单一的事件所充当, 是重点关注的对 象。通过对本体的解释可以辨别谣言不同的阶段, 为谣言的追根溯源, 进而对其 “辟谣” 提 供了一个有益的路径。两者合一完成了谣言事件的 “内核” 加 “保护膜” 的建构，由此充当 了谣言的内容。当借助外力带来的 “第一推动” 1)后, 谣言事件开始传播, 从而演变成“谣言”。

最后, 谣言具有被动的后知后觉性, 这也是谣言本身在传播过程中无法被证伪的原因。 即事件一旦被认定为谣言, 往往是因为与其相对应的 “非谣言” 的存在并被人广泛所知, 和 一定的官方及权威可信度高的机构所发出的 “辟谣”认定。谣言本身难以通过 “自圆其说” 的方式得到确认，它存在的本身就是与真实的信息对应。其虚假的本质需要通过对比的证明 来得到, 即谣言本身的 “假”之所以为假, 是与 “真” 相对应, 如无法证明 “真” 为真, “假” 亦无法被证伪。在 “假” 无法被证伪的基础上, “谣言” 就缺乏了存在的基础, 而是一种普通 信息被公众接受得以传播。由此拓展, 谣言在尚未传播, 仍处于谣言事件的最初状态时, 已 然不具备真实性，这是从 “谣言” 这一事实的结果来推断出其形成和传播的过程，最终追根 溯源到 “谣言” 虚假的源头。但是由于其后知后觉的特征, 在面临无法被证伪的实情下, 受 众对其的认知仍旧是一般信息, 无法出现对 “虚假” 这一负面符号的排斥和防御性反应。这 是传播过程中受众对谣言可信度的基础, 对其知晓范围的扩大提供了帮助。而谣言的被动性 则体现在, 谣言的存在是 “被推动” 的结果。即谣言的内容本身不会自己传播, 它需要借助 外力把内容首先释放到公众中间，正如对谣言定义所提及的，用这种 “第一推动” 把谣言的 内容塑造成一种與论或传闻。

\section{2. 谣言在澳门传播的特点和形式}

而谣言在一地的传播与发展, 除了满足自身的本质和基本要求外，也与时代变迁和当地 社会生态环境相适应。因此, 不同地域的谣言随着时间的变化也会出现些许的改变, 这是谣 言的地域化特征。它是基于谣言本质特征之上的衍生产物, 但又对谣言的产生和传播可以施 加相当大的影响, 因此不能被忽略。澳门本地谣言的传播与发展亦不例外。

探讨谣言在澳门的传播和发展轨迹, 要对澳门的社会环境初步了解。作为一个袖珍的独 立经济体，看似虽小的澳门在具有一般独立社会经济体的几乎所有特征之外，又具有自身的 独特性。

1.澳门的 “邻里社会” 性。澳门的社会生活体现着强烈的 “街坊” 性质, 即因为地域的 狭小，本地居民的居住相对集中，生产生活所面临的环境无法摆脱生活距离上的限制，体现 在社会关系中就是邻里特征尤为明显, 甚至是建立在一定的邻里关系之上。这种较为初级和 单纯的社会关系让谣言通过邻里间信任人的口口相传, 受众对其真实性的感知大为增强, 加 剧了谣言的影响和传播的生命力。

2.澳门的 “中西交融” 性。澳门在中西方交往史上长期承担桥梁的作用，它需要做到深 入交往的两端, 方能彰显其价值和意义。这也是澳门在政治和社会生态上长期 “内地融合” 与对外开放合一性的体现。即一方面以华人为主体居民体现的是中方的文化和价值观, 而过

\footnotetext{
(1) “第一推动” 是哲学学科中的概念, 论及的是推动世间万物最开始运动的一种不可知力。这里借用该概念想要表达的是谣
} 言的内容是需要通过外力来开始传播, 方可形成谣言。 
早的开埠让该地对西方文明报以接受的态度而不排斥。两者在澳门这一狭小的地域接触和碰 撞, 产生了大量与周遭完全不同的社会文化。因此, 谣言在澳门的传播也不得不体现这种中 外并举、华洋混杂的特征，这也是波谲云诡的国际关系的反映。

3. 澳门政治性质的多变性。澳门近五百年来的政治性质从完全由中央管辖的普通行政区, 到葡萄牙人的暂住地, 再到其租借地, 进而沦为葡萄牙的殖民地, 最终回归到中国的特别行 政区。其经历的政治性质变化之繁多为现今事实上认定的中国版图内之最, 体现在政局上的 变化则具有相当的特色。这种频繁的变化, 让谣言在澳门的传播也需要顺应这种复杂的趋势。 甚至不排除在一定的时期, 当政者为维持政局的稳定, 通过散布谣言来达到管制的目的。

直到 1999 年之后, 谣言在澳门回归后的传播与发展是随着葡萄牙人离开这一重大国际政 治与历史性事件, 辅以社会环境乃至澳门与内地关系、澳门与海外关系的剧烈变化和资讯媒 体的蓬勃演进而迅速展开而来。

除了前文所述澳门本地仍然具有的独特性外, 谣言在澳门的传播随着新时代本澳大环境 的变化也需要重新得到审视。这主要体现在:

1. 葡萄牙人离开后形成的短暂权力真空, 对于政府官员大量而迅速本地化而言, 在执政 能力上是一种考验, 至今仍然如此。

2.与香港类似, 回归后的澳门在国外, 特别是在个别西方国家看来, 是可以充当掣肘中 国的手段之一。(2)

3.澳门本土资讯媒体薄弱的劣势, 使之较难获得话语权。本地在电子、互联网等成体系 依赖域外新兴媒介的局面至今仍然没有得到改观, 常会受制于人。官方对其的态度更多倾向 于以“不管”代替“支持”的懒政式思维。

4.澳门与祖国内地的趋同在回归后更趋明显。以往被葡萄牙 “代管”的政治隔阂在回归 后已不存在。同时由于经济发展和综合实力的增强, 中国内地的强势影响力伴随着中央政府 诸多惠及澳门的政策之施行, 让澳门与内地的趋同性大大增强。此外澳门本地相较于香港多 元的政治生态而言，较为单一的政治格局从另外一面加强了这种趋势。

5. 澳门地理位置的狭小体现在政府层面就是管制范围的局限性，这是其劣势。特别是在 当今资讯科技空前发达的时代, 澳门对外交往的频繁性, 使得众多需要管制的事项往往会超 出执政当局的管辖范围而难以实施。但是管辖区域的狭小在政策施行方面又相对单一, 其投 放的精准程度和执行效果在官方执政能力不断提高的前提下能够获得较好效果, 这是其治理 层面的优势。

2017 年 “天鸽”台风从澳门过境之后带来的巨大灾难, 引发了众多广为流传的谣言, 其 中 “解放军澳门杀人” 是近年澳门出现的最为著名, 影响力和破坏性最大的谣言之一, 引起 了从政府到民间多方面的强烈关注。

从社交媒体上流传的言论可以看到, 以 “解放军係澳门杀人啦” 作为标题的谣言, 以及 两段内容的本体均为 “解放军”, 这是该谣言的核心。对其的解释从 “杀人” 作为标题吸引眼 球，并在叙述中使用 “抢劫”、“殴打市民”, 直到 “殴打致死” 的字样层层升级来突出解放军 “杀人” 行为的程度, 结合诸多辅助性的描述, 谣言的散布者力图渲染出一种恐怖的气氛。 它们是核心的外延, 凸显了对谣言本体和整个谣言的保护, 使其能够持续不断的传播。这种 典型谣言的出现和传播并非是孤立的个体，它是谣言的普遍性和特殊性综合的产物。

首先标题就十分吸引眼球。本应作为抵御外敌和协助救灾的国家武装力量, 在高度自治 的澳门“杀人”, 本身就能引起强烈关注。特别是在澳门受灾急需救济的关键时刻——“杀人”, 严重违背公众的基本理念以及对其的普遍印象, 甚至扭曲了对其的认知, 自然会受到特别关

(1) 国家主席习近平 2018 年 01 月 05 日在 “学习贯彻党的十九大精神研讨班开班式上发表的重要讲话” 中对周边局势使用的 也是 “波谲云诡” 这个词。此外, 前总理朱F基在一次答记者问上对国际关系的描述也用到该词。可见, 用 “波谲云诡” 来 形容国际关系一直是较为贴切的用词。

(2) 外交部发言人就欧盟发表 2016 年度港澳特区年度报告答记者问: http://www.fmprc.gov.cn/ce/cohk/chn/gsxw/t1457897.ht m。 
注。这是谣言散布者首要达到的目的, 力图让更多的人关注。此外，标题可以直接概括为 “解 放军澳门杀人”, 它本来就是一个被缩减后的谣言, 令其传播更广泛速度更快, 迎合了当下快 餐文化的特点和需求。以至于诸多内地媒体报道该新闻时，均以其为关键字甚至作为标题。(1)

其次互联网所具有的开放性和信息传播的广泛性被造谣者利用。谣言被公开发布于各类 著名论坛和影响力大的社交网络上, 目的就是为了传播。而互联网和社交媒体的传播特性得 以叠加使用, 故意扩散该谣言的目的与意图十分明显。其始作俑者和散布者均为有意通过这 种本质上是 “被推动” 的方式, 来传播和扩大谣言影响力。不论是否为谣言的源头, 从广泛 引用的一位谣言内容提供者的账户名 “LoveUKLoveHK38”, 也不难推断其政治倾向。它亦为谣 言散布的动机提供了一个佐证。

此外，该谣言只是简单表述了事情的发生，没有进一步证实事件的真实性，具有谣言典 型的模糊性特征。而网络上传播的相同内容的谣言, 有的会加入类似 “亲眼所见” 来夯实真 实性, 却仍然无法提供令人信服的第三方证据, 这是谣言模糊性难以界定的方面。此外, 消 息发布者均为匿名, 且澳门保安司司长黄少泽证实造谣者是从美国把信息发至香港, 其间途 经多个国家的服务器, [2]来规避追踪, 则说明了谣言难以纠责的程度。这又体现了澳门处理 类似谣言的管理难度, 造谣源头来自澳门域外的美国, 除了警方 “连日带走了 4 名嫌疑人, 他们涉嫌传播 “停车场发现 5 具遗体” ” [3]的谣言外, 并无更多消息指向管理机构的进一步举 措。

最后官方的 “辟谣” (2) 则被动证明了 “解放军澳门杀人”系谣言的事实，无论其在开始 传播之时的真实性与否。把这一事件与同时出现的 “停车场发现 5 具遗体” 的谣言结合起来 看，被官方认定为 “对市民影响程度非常高，以致人心惶惶” [4]，一方面是对谣言造成结果 的说明, 也是众多造谣者和传谣者需要达到的目标。而引起官方 “严厉谴责” 的原因, 说明 了事件的严重程度到了不得不 “辟谣” 的时候, 这是造传谣者力求达到的目标, 亦为该谣言 的价值特征。另一方面又说明市民对其的接受程度之高, 才会达到 “影响程度非常高” 的现 实, 造成人心惶惶的效果。此间具有澳门特色的 “邻里社会” 性做出了很大的作用。谣言通 过网络精准投放到澳门, 接触到它们的受众通过线下地推的方式口口相传, 基于 “邻里社会” 人与人之间的可信度则加剧了谣言的传播效果, 使其的生命力得以加强, 进而扩大了谣言的 影响。因此, 让受众信任和接受, 是谣言传播的条件。而一旦被认定为谣言, 或被受众认识 到其传递的信息为虚假, 谣言的传播即终止, 其生命得以终结, 但造成的连环影响会随着程 度的高低持续存在一段时间。

\section{3. 建议与对策}

鉴于谣言的基本特征并结合澳门本地特色，建议采取如下对策来切实有效的防范和化解 谣言的传播和对本地的影响。

重视社区的建设和管理工作, 加大社区志愿者的培训, 借助志愿者能够深入社区的优势, 来充当谣言 “粉碎机” 的作用。尽可能的采取面对面的方法来减少甚至杜绝谣言在人际间的 传播。同时加强针对性的教育, 强化居民辨识虚假信息的能力, 因为 “如果公众有很强的批 判能力, 能够识别谣言的假像, 那么谣言也不可能传播开来” [5]。以总体上 60 万人的本地常 住居民为限，以特区政府强大的财力作为支撑，这并非是一件难以完成的期许。

由于澳门本地的谣言多为输入性，且澳门的媒体管制力度相较于祖国内地而言更弱，但 是相比香港管制力度更强。因此, 澳门在对海外输入性谣言的管制工作方面, 更需要精确的 把握好反制时机，力求以更小代价获得更大收益。

由于本身管辖范围的限制, 对于多出现输入性谣言的澳门, 追根溯源的难度已然很大, 对其采取一定的包括惩罚措施在内的实际手段就显得鞭长莫及。因此着重做好谣言在本地的

\footnotetext{
(1) 在中国的搜索引擎中输入关键字 “澳门” 和 “谣言”, 可以发现众多此类新闻的标题。

(2) (澳门) 司法警察局澄清网上谣言: http://portal.gov.mo/web/guest/info_detail?infoid=2390764。
} 
次级传播就显得尤为重要, 这种管理的成本相对较为低廉。

相较于内地庞大的社会群体而言, 属于微型社会的澳门对于谣言的管控更有利于采取具体措 施，这是其优势。因此，提高官方层面整体的运营和管制能力，进而延伸到政府的执政能力 层面, 是有效抑制谣言传播和危害的最终手段, 也是考验每个澳门上至官方下至基层人员综 合素质和能力的保障性手段。

\section{References}

[1] http://blog.sina.com.cn/s/blog_b7d2fcd50102v2ed.html.

[2] http://news.sina.com.cn/o/2017-08-31/doc-ifykpzey3361922.shtml.

[3] Source above.

[4] Source above.

[5] C. Manli and Q. Yunxia editors in chief, Communication Dictionary, Xinhua Publishing House: Beijing, China, 2012. 